$\Im D$ Optical Spectroscopic Methods in Astronomy

ASP Conference Series, Vol. 71, 1995

G. Comte, M. Marcelin, eds.

\title{
Michelson-Spectro-Interferometry (MSI) of Be stars envelopes with the GI2T Interferometer
}

\author{
Ph. Stee, D. Bonneau, P. Lawson, F. Morand, D. Mourard, \\ I. Tallon, and F. Vakili \\ Observatoire de la Côte d'Azur \\ 2130 route de l'Observatoire, Caussols \\ $06\{60$ St Vallier de Thiey, France
}

\begin{abstract}
The GI2T is an optical long-baseline Michelson interferometer which analyses dispersed stellar fringes in the multi-speckle mode with a spectral resolution of one Angstrom while the spatial resolution is about one milliarcsecond. This makes the GI2T a powerful instrument able to perform MSI of extended sources like Be stars or shell stars. In order to interpret these data we have developed a latitude dependent radiative wind model for Be stars. This numerical code enables us to compare directly computed 2D maps in some Balmer lines ( $H \alpha$ and $H \beta$ ) with high angular resolution data of some Be stars.
\end{abstract}

\section{The GI2T interferometer}

The Grand Interféromètre à 2 Télescopes (GI2T) is a long-baseline Michelson interferometer located on the Plateau de Calern above the town of St. Vallier de Thiey in southern France. With baselines as large as $70 \mathrm{~m}$ and apertures of 1.5 $m$ in diameter, it provides a spatial resolution of up to two milliarcseconds for the study of astrophysical phenomena at optical wavelengths.The GI2T is the only stellar interferometer that records dispersed stellar fringes in a multi-speckle mode, having a maximum spectral resolution of $1.0 \AA$. The fringes appear across each speckle in the image and have a spacing dependent on the separation of the remapped pupils, similar to those produced by a Young's double-slit experiment. Image slicing is used to isolate a vertical band of speckles within the seeing disk, and this slice is dispersed with the orientation of the fringes perpendicular to the direction of dispersion. The ability to obtain simultaneous visibility measurements at selected wavelength channels is a key feature of the GI2T.

We record short exposure images of the fringes using an intensified CCD camera with a frame time of 20 ms to freeze the effects of atmospheric turbulence. From these frames the visibility is measured at each wavelength by performing an autocorrelation of the fringes in the direction of the image slice. The ensemble average of the autocorrelations is then Fourier transformed to yield a spatial power spectrum, from which the visibility is calculated. We have developed a new estimator for visibility that is in principle independent of the seeing conditions (Mourard et al. 1994). Of course, the same data can be used 


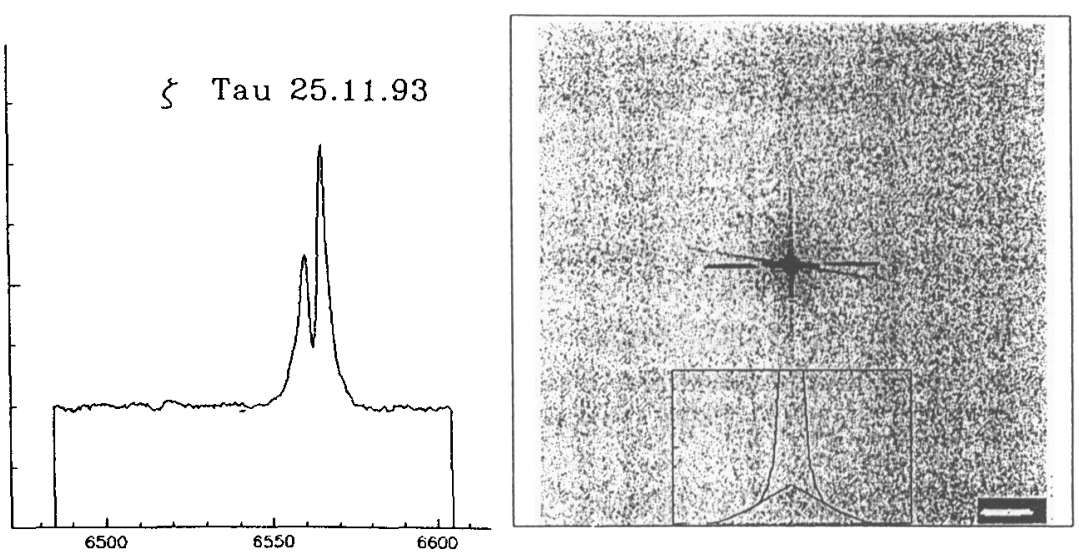

Figure 1. The $\mathrm{H} \alpha$ line profile of the star $\zeta$ Tau calibrated in wavelength (left). Also shown is a spatial power spectrum (right) illustrating the features arising from the speckles and fringes.

in long exposures to derive stellar spectra, also used in the analysis. An example of this is shown in Figure 1.

\section{Measurements of Be stars}

Although we can use the interferometer to observe various stellar sources, be they single or binary, the envelopes of Be stars have been our principle object of study. Their photospheres, which are typically unresolved by the interferometer, are sources of continuum emission that can be used to calibrate visibility measurement made at emission lines. Emission from the Balmer lines originates in the extended hydrogen envelope which surrounds the stars and may be mapped in detail, including its morphology and velocity distribution.

A two-telescope interferometer is only sensitive to one-dimensional information of the source at any one time. However, by earth-rotation synthesis we can map the object at many different angles and study the asymmetry of the circumstellar matter. More interestingly we can follow the relative phase of the visibility at different Doppler shifts in a broadened emission line. The measured phase offsets are related, at first order, to differences in the position of the source as a function of wavelength. This is shown using a model distribution illustrated in Figure 2. This can yield details of the expansion velocity relative to the rotational velocity, and more directly the direction of rotation of the star.

\section{A latitude-dependent radiative wind model for Be stars}

We have developed a latitude-dependent radiative wind model for Be stars which clearly shows that the brightness distribution of the circumstellar envelope is strongly dependent on the central observation wavelength and bandwidth 

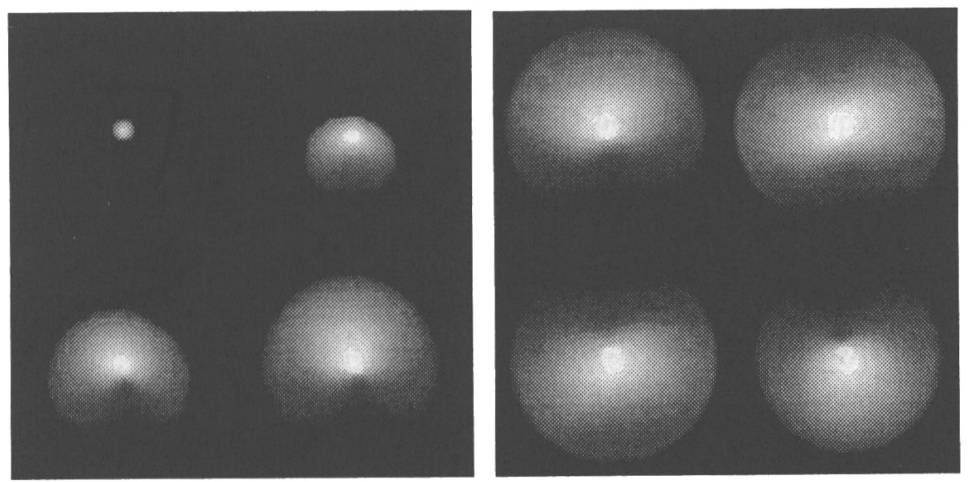

Figure 2. $\mathrm{H} \beta$ intensity maps at various Doppler-shifts across the $\mathrm{H} \boldsymbol{\beta}$ line profile computed from our model. From $4835 \AA$ (upper left) to $4870 \AA$ (lower right) by steps of $5 \AA$.
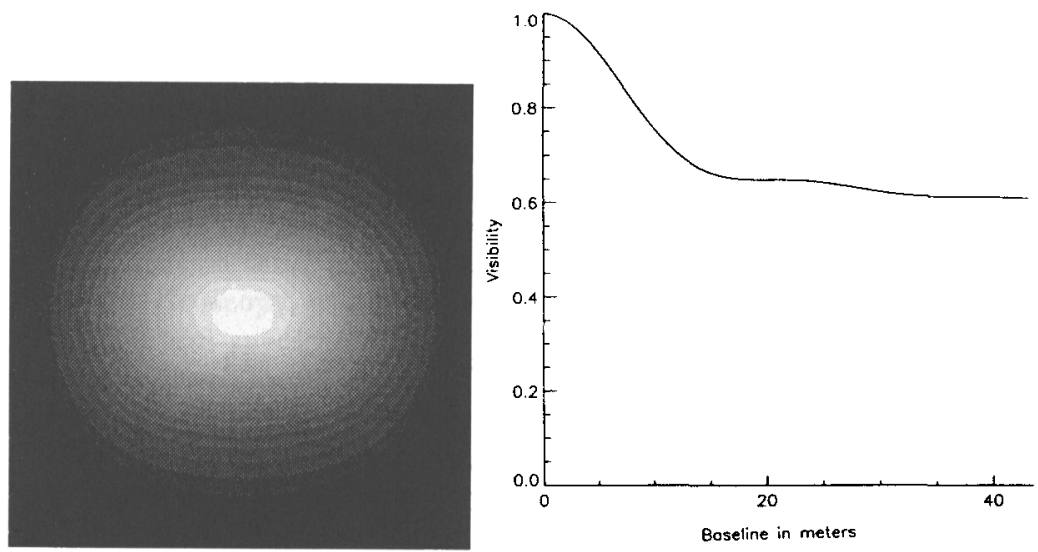

Figure 3. H $\alpha$ intensity map with a bandwidth of $20 \AA$ at an inclination angle of $45^{\circ}$ computed from our model (left) and the corresponding theoretical visibility curve as a function of baseline for the GI2T (right). 
(Araújo et al. 1989, 1993; Stee and Araújo 1993). Our software for hydrody. namic modeling enables us to consider the effects of a viscous force in the azimuthal component of the momentum equations. We use the same line force as used by Friend and Abbott (1986), but introduce a varying contribution of thin and thick lines from pole to equator by adopting latitude-dependent radiative parameters. We have derived velocity fields and density relationships from the hydrodynamic equations and used them to solve for the conditions of statistical equilibrium. By adopting the Sobolev approximation, we have calculated the electron density and hydrogen level populations throughout the envelope.

We obtained asymmetric double-peaked $\mathrm{H} \alpha$ and $\mathrm{H} \beta$ emission profiles from our rotating and expanding wind model. Our computation takes into account the portion of the envelope occulted by the stellar disc and the absorption of the photospheric line. We have also computed intensity maps of the circumstellar envelope in the lines and continuum. As shown in Figure 3, our code enables us to directly compare simulated maps at Balmer lines with high angular resolution data of Be stars. A comparison between our predictions and interferometric measurements of $\gamma$ Cas is now in preparation.

Long baseline optical interferometry, along with the more traditional methods of photometry, polarimetry, and spectroscopy, is a very powerful tool for understanding the physics of extended atmospheres around early type stars.

\section{Discussion}

Chalabaev: Could you comment on recent data from the GI2T?

Stee: We have carried out extensive observations of the Be star $\gamma$ Cas during the autumn of 1993. We have spectro-interferometric data covering two spectral bands centred on $\mathrm{H} \alpha$ and $\mathrm{H} \beta$, with a resolution ranging from 3 to 10 milliarcseconds. Preliminary analysis confirms that the envelope in these emission lines is resolved and that $\gamma$ Cas is partially resolved in the continuum. We hope to soon publish a comparison of these observations and our model.

\section{References}

Araújo, F.X., Freitas Pacheco, J.A. 1989, MNRAS, 241, 543

Araújo, F.X., Freitas Pacheco, J.A., Petrini, D. 1993, MNRAS, 267, 501

Friend, D.B., Abbott, D.C. 1986, ApJ, 311, 701

Mourard, D., Bosc, I., Blazit, A., Bonneau, D.,Merlin, G., Morand, F., Vakili, F., Labeyrie, A. 1994, A\&A, 283, 705

Stee, Ph. and Araújo, F.X. 1994, A\&A, in press 